\title{
3 Research Square \\ Modeling the Risk of Radiation Pneumonitis in Esophageal Squamous Cell Carcinoma Treated with Definitive Chemoradiotherapy
}

\section{Kaiqi Lan}

Sun Yat-sen University Cancer Center

Cheng Xu

Sun Yat-sen University Cancer Center

Shiliang Liu

Sun Yat-sen University Cancer Center

Jinhan Zhu

Sun Yat-sen University Cancer Center

\section{Yadi Yang}

Sun Yat-sen University Cancer Center

\section{Li Zhang}

Sun Yat-sen University Cancer Center

Mian Xi ( $\nabla$ ximian@sysucc.org.cn )

Sun Yat-sen University Cancer Center

\section{Research}

Keywords: Esophageal squamous cell carcinoma, definitive chemoradiotherapy, radiation pneumonitis, nomogram, prediction model

Posted Date: December 8th, 2020

DOI: https://doi.org/10.21203/rs.3.rs-120618/v1

License: (c) (1) This work is licensed under a Creative Commons Attribution 4.0 International License. Read Full License 


\section{Abstract}

Purpose: To develop and validate a nomogram for the prediction of symptomatic radiation pneumonitis (RP) in patients with esophageal squamous cell carcinoma (ESCC) who received definitive concurrent chemoradiotherapy.

Methods: Clinical factors, dose-volume histogram parameters, and pulmonary function parameters were collected from 402 ESCC patients between 2010 and 2017, including 321 patients in the primary cohort and 81 in the validation cohort. The end-point was the occurrence of symptomatic RP (grade $\geq 2$ ) within the first 12 months after radiotherapy. Univariate and multivariate logistic regression analyses were applied to evaluate the predictive value of each factor for RP. A prediction model was generated in the primary cohort, which was internally validated to assess its performance.

Results: In the primary cohort, 31 patients (9.7\%) experienced symptomatic RP. Based on logistic regression model, patients with larger planning target volumes (PTVs) or higher lung $\mathrm{V}_{20}$ had a higher predictive risk of RP, whereas the overall risk was substantially higher for three-dimensional conformal radiotherapy (3DCRT) than intensity-modulated radiotherapy. On multivariate analysis, independent predictive factors for RP were smoking history $(P=0.018)$, radiotherapy modality $(P<0.001)$, PTV $(P=0.014)$, and lung $\mathrm{V}_{20}(P=0.002)$, which were incorporated into the nomogram. The areas under the receiver operating characteristic curve of the nomogram in the primary and validation cohorts were 0.772 and 0.900 , respectively, which were superior to each predictor alone.

Conclusions: Non-smoking status, 3DCRT, lung $\mathrm{V}_{20}(>27.5 \%)$, and PTV ( $\left.\geq 713.0 \mathrm{cc}\right)$ were significantly associated with a higher risk of RP. A nomogram was built with satisfactory prediction ability.

\section{Introduction}

Esophageal cancer (EC) is one of the leading causes of cancer-related death worldwide, with an estimated 508,585 deaths yearly [1]. Definitive chemoradiotherapy (CRT) is the standard care in the management of unresectable EC [2,3]. Radiation pneumonitis (RP) is one of the major complications after thoracic irradiation. Despite low mortality, RP can result in respiratory insufficiency, seriously affect patients' quality of life, influence the completion of radiotherapy and following treatment, and even reduce the curative effect, particularly for patients with symptomatic RP [4]. Although the survival benefit of concurrent CRT over radiotherapy alone is evident, the former has been demonstrated to be correlated with an increased risk of RP[5]. Therefore, decreasing the incidence of RP is of critical clinical significance for EC patients who received concurrent CRT.

An increasing number of studies have investigated predictors for RP [4-12]. Various risk factors, including clinical characteristics, dosimetric parameters, treatment-related variables, as well as serum biomarkers have been reported to be associated with the occurrence of RP [4-12]. However, the majority of these reports focused on patients with lung cancer, and data regarding EC patients undergoing definitive CRT is limited. Considering the differences in baseline pulmonary function and in the level of 
radiation dose between lung cancer and EC patients, it is unreasonable to extrapolate risk factors for RP in lung cancer to EC. On the other hand, despite the significantly increasing use of intensity-modulated radiotherapy (IMRT) in recent years, its impact on RP remains unclear in EC.

Therefore, the purpose of study was to investigate the associations between clinical factors, dose-volume histogram (DVH) parameters, as well as pulmonary function parameters, and the risk of symptomatic RP in patients with esophageal squamous cell carcinoma (ESCC) who received definitive concurrent CRT. Then a nomogram model predicting RP was developed and validated to guide clinical decision-making.

\section{Patients And Methods}

\section{Patients}

All consecutive ESCC patients who underwent definitive CRT at our institution from January 2010 through October 2017 were retrospectively analyzed. Inclusion criteria were defined: pathologic confirmation of stage I-IVa ESCC according to the 8th TNM staging system of the American Joint Committee on Cancer [13], receipt of concurrent CRT with curative intent, DVH data retrievable from treatment planning system, radiographic images and symptom assessments available to evaluate the occurrence of RP, and the availability of pulmonary function tests prior to CRT. Patients with prior or concomitant malignancy, those with previous thoracic radiotherapy or surgery, and those with incomplete records were excluded. A total of 402 eligible ESCC patients were included, including 321 patients in the primary cohort and 81 patients in the validation cohort based on a randomization ratio of 4:1. This study was approved by the Institutional Review Boards of Sun Yat-sen University Cancer Center (No. B2019-010-01). Since this was a retrospective analysis of routine data, we requested, and were granted, a waiver of individual informed consent from the ethics committee. Patient records/information was anonymized and deidentified before analysis.

\section{Data Collection}

The following data were collected for each patient from the medical records: medical history, patient and tumor characteristics, treatment information, radiographic images, and symptom assessments. Pulmonary function parameters included forced expiratory volume in the first second and diffusing capacity for carbon monoxide. DVH parameters were extracted from the treatment planning system: planning target volume (PTV), total lung volume (TLV), mean lung dose (MLD), and the percentage of lung volume receiving more than $x \mathrm{~Gy}\left(\mathrm{~V}_{\mathrm{x}}\right)$, ranging from 5 to $30 \mathrm{~Gy}$ in increments of $5 \mathrm{~Gy}$.

\section{Treatment}

A fraction of patients received 1 to 4 cycles of induction chemotherapy prior to CRT, and all patients received concurrent platinum- or taxane-based chemotherapy during radiotherapy, as determined by the multidisciplinary team. For radiotherapy, gross tumor volume (GTV) encompassed the primary tumor and involved lymph nodes. Clinical target volume (CTV) was defined as the GTV plus a 3-cm margin in proximal and distal direction and a radial margin of 0.5 to $1.0 \mathrm{~cm}$. PTV was defined as CTV plus a 0.5- 
$0.8 \mathrm{~cm}$ margin to account for setup uncertainty. Patients were treated with three-dimensional conformal radiotherapy (3DCRT) or IMRT to deliver the prescribed dose of 50-70 Gy in 25-35 fractions. Dose constraints for normal tissues were defined as follows: the maximum dose of spinal cord < $45 \mathrm{~Gy}$; lung $\mathrm{V}_{5}<65 \%, \mathrm{~V}_{20}<30 \%$, and MLD $<17$ Gy; heart $\mathrm{V}_{30}<40 \%$ and mean dose $<28 \mathrm{~Gy}$.

\section{Evaluation of RP and Follow-up}

Patients were followed 1 month after CRT, then every 3 months during the first 2 years, every 6 months for the next 3 years, and then annually. Chest computed tomography was performed at each visit. The relevant clinical symptoms were recorded by the treating physician in medical records.

The end-point for this study was the occurrence of symptomatic RP (grade $\geq 2$ ) within the first 12 months after radiotherapy, which was diagnosed by clinical symptoms, chest imaging, and evidence of medication and treatment in the medical records. RP was graded based on National Cancer Institute Common Toxicity Criteria for Adverse Events version 4.0 (CTCAE v4.0).

\section{Statistical Analysis and Modeling}

Age, tumor length, radiation dose, and pulmonary function parameters were grouped by the median value as cut-offs. Univariate and multivariate logistic regression models were conducted to analyze possible predictors for RP. Variables with $P \leq 0.15$ in the univariate analysis were assessed in multivariate analysis (backward stepwise). Prior to multivariate analysis, Spearman rank correlation analyses were performed to check multicollinearity between factors.

Factors with significant predictive value in multivariate analysis were used to build the nomogram in the primary cohort. Then the nomogram was validated in the validation cohort. The performance of the nomogram was assessed by the area under the receiver operating characteristic (ROC) curve (AUC), calibration curve using 1000 bootstrap resamples, and decision curve analysis (DCA). Calibration curve was generated to compare the predicted with the observed probability of RP. DCA was employed to evaluate the clinical usefulness of the nomogram. Moreover, the optimal cut-offs of the continuous parameters in the nomogram was calculated using the ROC curves. Statistical analyses were performed using SPSS 22.0 software (SPSS Inc., Chicago, IL) and R software (version 3.4.3). A $P$ value $<0.05$ was considered to be statistically significant.

\section{Results}

\section{Patient Characteristics}

Patient and treatment characteristics of 402 ESCC patients who met the inclusion criteria are summarized in Table 1. For the primary cohort $(n=321)$, the median age was 59 years (range, 2973 years) and the majority of the tumors were located in the upper/middle esophagus (86.0\%). Of them, 218 patients (67.9\%) were current or former smokers, and COPD accounted for only 5.6\%. A total of 195 patients $(60.7 \%)$ received induction chemotherapy prior to concurrent CRT. The majority of patients 
(78.2\%) were treated with IMRT, and the rest with 3DCRT, with the median radiation dose of $60.0 \mathrm{~Gy}$ (range, 36-70 Gy). A small fraction of patients $(5.3 \%$ ) received a dose of $<50.0$ Gy owing to treatmentrelated toxicity. As for DVH parameters, the median PTV was $683.1 \mathrm{cc}$ (range, 253.0-1566.0 cc) and median TLV was $3240.3 \mathrm{cc}$ (range, 1697.3-7961.9.0 cc). MLD ranged from 4.9 to $21.5 \mathrm{~Gy}$, with a median of $14.8 \mathrm{~Gy}$. Median $\mathrm{V}_{5}, \mathrm{~V}_{10}, \mathrm{~V}_{15}, \mathrm{~V}_{20}, \mathrm{~V}_{25}$, and $\mathrm{V}_{30}$ of lung were $70.1 \%, 49.5 \%, 36.2 \%, 27.3 \%, 20.6 \%$, and $14.8 \%$, respectively. 
Table 1

Patient characteristics.

\begin{tabular}{|c|c|c|c|c|}
\hline Characteristic & Total $(n=402), \%$ & $\begin{array}{l}\text { Primary cohort (n } \\
=321), \%\end{array}$ & $\begin{array}{l}\text { Validation cohort } \\
(n=81), \%\end{array}$ & $\begin{array}{l}P \\
\text { value }\end{array}$ \\
\hline \multicolumn{5}{|l|}{ Clinical factors } \\
\hline $\begin{array}{l}\text { Age (years), Median } \\
\text { (range) }\end{array}$ & $59(29-73)$ & $59(29-73)$ & $60(40-71)$ & 0.087 \\
\hline \multicolumn{4}{|l|}{ Sex } & 0.004 \\
\hline Male & $294(73.1)$ & $245(76.3)$ & $49(60.5)$ & \\
\hline Female & $108(26.9)$ & $76(23.7)$ & $32(39.5)$ & \\
\hline \multicolumn{4}{|l|}{ Smoking history } & 0.670 \\
\hline Yes & $270(67.2)$ & $218(67.9)$ & $53(65.4)$ & \\
\hline No & $132(32.8)$ & $103(32.1)$ & $28(34.6)$ & \\
\hline \multicolumn{4}{|l|}{ COPD } & 0.845 \\
\hline Yes & $23(5.7)$ & $18(5.6)$ & $5(6.2)$ & \\
\hline No & 379 (94.3) & $303(94.4)$ & $76(93.8)$ & \\
\hline \multicolumn{4}{|c|}{ ECOG performance status } & 0.893 \\
\hline 0 & $268(66.7)$ & $215(67.0)$ & $53(65.4)$ & \\
\hline $1-2$ & $134(33.3)$ & $106(33.0)$ & $28(34.6)$ & \\
\hline \multicolumn{4}{|l|}{ Weight loss } & 0.790 \\
\hline$<10 \%$ & 317 (78.9) & $254(79.1)$ & $63(77.8)$ & \\
\hline$\geq 10 \%$ & $85(21.1)$ & $67(20.9)$ & $18(22.2)$ & \\
\hline \multicolumn{4}{|l|}{ Tumor location } & 0.045 \\
\hline Upper & 159 (39.5) & $127(39.6)$ & $32(39.5)$ & \\
\hline Middle & $178(44.3)$ & 149 (46.4) & $29(35.8)$ & \\
\hline Distal & $65(16.2)$ & $45(14.0)$ & $20(24.7)$ & \\
\hline \multicolumn{4}{|l|}{ Primary tumor length } & 0.358 \\
\hline \multicolumn{5}{|c|}{$\begin{array}{l}\text { Abbreviations: COPD, chronic obstructive pulmonary disease; ECOG, Eastern Cooperative Oncology } \\
\text { Group; } 3 D C R T \text {, three-dimensional conformal radiation therapy; IMRT, intensity-modulated radiation } \\
\text { therapy; } V_{x} \text { : percentage of the total lung volume receiving more than x Gy; IQR, interquartile range; } \\
\text { MLD, mean lung dose; PTV, planning target volume; TLV, total lung volume; FEV1: forced expiratory } \\
\text { volume in the first second; DLCO, diffusing capacity for carbon monoxide. }\end{array}$} \\
\hline \multicolumn{5}{|c|}{ *: Single agent chemotherapy during radiotherapy (taxane or platinum). } \\
\hline
\end{tabular}




\begin{tabular}{|c|c|c|c|c|}
\hline Characteristic & Total $(n=402), \%$ & $\begin{array}{l}\text { Primary cohort }(\mathrm{n} \\
=321), \%\end{array}$ & $\begin{array}{l}\text { Validation cohort } \\
(n=81), \%\end{array}$ & $\begin{array}{l}P \\
\text { value }\end{array}$ \\
\hline$<6 \mathrm{~cm}$ & $230(57.2)$ & $180(56.1)$ & $50(61.7)$ & \\
\hline$\geq 6 \mathrm{~cm}$ & $172(42.8)$ & $141(43.9)$ & $31(38.3)$ & \\
\hline Clinical TNM stage & & & & 0.851 \\
\hline I/II & $42(10.5)$ & $34(10.6)$ & $8(9.9)$ & \\
\hline III/IVa & $360(89.6)$ & $287(89.4)$ & $73(90.1)$ & \\
\hline Induction chemotherapy & & & & 0.655 \\
\hline Yes & $242(60.2)$ & $195(60.7)$ & $47(58.0)$ & \\
\hline No & $160(39.8)$ & $126(39.3)$ & $34(42.0)$ & \\
\hline Concurrent chemotherapy & & & & / \\
\hline Yes & $402(100.0)$ & $321(100.0)$ & $81(100.0)$ & \\
\hline Chemotherapy regimen & & & & 0.009 \\
\hline Cisplatin/taxane & $232(57.7)$ & 173 (53.9) & $59(72.8)$ & \\
\hline Cisplatin/fluorouracil & 78 (19.4) & $68(21.2)$ & $10(12.3)$ & \\
\hline Other ${ }^{\star}$ & $92(22.9)$ & $80(24.9)$ & $12(14.8)$ & \\
\hline Radiation dose (Gy) & & & & 0.617 \\
\hline$\leq 60$ & $299(74.4)$ & $237(73.8)$ & $62(76.5)$ & \\
\hline$>60$ & $103(25.6)$ & $84(26.2)$ & $19(23.5)$ & \\
\hline Radiotherapy modality & & & & 0.137 \\
\hline 3DCRT & $92(22.9)$ & $70(21.8)$ & $24(29.6)$ & \\
\hline IMRT & $310(77.1)$ & 251 (78.2) & $57(70.4)$ & \\
\hline \multicolumn{5}{|l|}{ Dosimetric parameters } \\
\hline $\mathrm{V}_{5}(\%)$, Median (IQR) & $69.8(60.6-80.9)$ & $70.1(60.8-81.3)$ & $68.2(57.3-75.5)$ & 0.132 \\
\hline $\mathrm{V}_{10}(\%)$, Median (IQR) & $49.3(42.3-56.1)$ & $49.5(42.4-57.2)$ & $48.9(41.4-53.7)$ & 0.260 \\
\hline \multicolumn{5}{|c|}{$\begin{array}{l}\text { Abbreviations: COPD, chronic obstructive pulmonary disease; ECOG, Eastern Cooperative Oncology } \\
\text { Group; 3DCRT, three-dimensional conformal radiation therapy; IMRT, intensity-modulated radiation } \\
\text { therapy; } V_{x} \text { : percentage of the total lung volume receiving more than x Gy; IQR, interquartile range; } \\
\text { MLD, mean lung dose; PTV, planning target volume; TLV, total lung volume; FEV1: forced expiratory } \\
\text { volume in the first second; DLCO, diffusing capacity for carbon monoxide. }\end{array}$} \\
\hline
\end{tabular}




\begin{tabular}{|c|c|c|c|c|}
\hline Characteristic & Total $(n=402), \%$ & $\begin{array}{l}\text { Primary cohort (n } \\
=321), \%\end{array}$ & $\begin{array}{l}\text { Validation cohort } \\
(n=81), \%\end{array}$ & $\begin{array}{l}P \\
\text { value }\end{array}$ \\
\hline $\mathrm{V}_{15}(\%)$, Median (IQR) & $36.4(31.3-40.9)$ & $36.2(31.5-41.2)$ & $36.5(30.5-39.9)$ & 0.377 \\
\hline $\mathrm{V}_{20}(\%)$, Median (IQR) & $27.2(22.3-30.2)$ & $27.3(22.8-30.5)$ & $25.9(20.8-29.6)$ & 0.058 \\
\hline $\mathrm{V}_{25}(\%)$, Median (IQR) & $20.3(16.0-23.0)$ & $20.6(16.3-22.9)$ & $19.2(13.7-23.1)$ & 0.127 \\
\hline $\mathrm{V}_{30}(\%)$, Median (IQR) & $\begin{array}{l}14.87(11.0- \\
17.7)\end{array}$ & $14.8(11.5-17.7)$ & $13.8(8.6-18.3)$ & 0.133 \\
\hline MLD (Gy), Median (IQR) & $14.8(12.6-16.6)$ & $14.8(12.9-16.7)$ & $14.6(12.0-16.1)$ & 0.121 \\
\hline PTV (cc), Median (IQR) & $\begin{array}{l}663.4(520.9- \\
816.6)\end{array}$ & $\begin{array}{l}683.1(540.2- \\
826.6)\end{array}$ & $\begin{array}{l}576.7(447.4- \\
793.5)\end{array}$ & 0.004 \\
\hline TLV (cc), Median (IQR) & $\begin{array}{l}3207.9(2698.1- \\
3884.5)\end{array}$ & $\begin{array}{l}3240.3(2704.1- \\
3897.0)\end{array}$ & $\begin{array}{l}3177.0(2689.7- \\
3833.1)\end{array}$ & 0.394 \\
\hline \multicolumn{5}{|l|}{$\begin{array}{l}\text { Pulmonary function } \\
\text { parameters }\end{array}$} \\
\hline FEV1 (L), Median (IQR) & $2.7(2.2-3.1)$ & $2.7(2.3-3.1)$ & $2.6(2.2-3.0)$ & 0.492 \\
\hline FEV1 (\%), Median (IQR) & $\begin{array}{l}93.5(76.0- \\
103.8)\end{array}$ & $93.6(78.0-104.8)$ & $93.2(75.3-102.0)$ & 0.473 \\
\hline $\begin{array}{l}\text { DLCO }(\mathrm{ml} / \mathrm{mmHg} / \mathrm{min}) \\
\text { Median (IQR) }\end{array}$ & $24.4(20.7-27.5)$ & $24.2(20.8-27.7)$ & $25.0(20.7-27.1)$ & 0.412 \\
\hline \multicolumn{5}{|c|}{$\begin{array}{l}\text { Abbreviations: COPD, chronic obstructive pulmonary disease; ECOG, Eastern Cooperative Oncology } \\
\text { Group; } 3 D C R T \text {, three-dimensional conformal radiation therapy; IMRT, intensity-modulated radiation } \\
\text { therapy; } V_{x} \text { : percentage of the total lung volume receiving more than x Gy; IQR, interquartile range; } \\
\text { MLD, mean lung dose; PTV, planning target volume; TLV, total lung volume; FEV1: forced expiratory } \\
\text { volume in the first second; DLCO, diffusing capacity for carbon monoxide. }\end{array}$} \\
\hline \multicolumn{5}{|c|}{ *: Single agent chemotherapy during radiotherapy (taxane or platinum). } \\
\hline
\end{tabular}

The median follow-up time was 21.5 months (range, 3.5-108.8 months) for the primary cohort. During follow-up, 159 patients (49.5\%) had grade 1 RP, 27 (8.4\%) had grade 2 RP, $3(0.9 \%)$ had grade 3 RP, 1 $(0.3 \%)$ had grade 4 RP, and no patients had grade 5 RP. In total, 31 patients $(9.7 \%)$ experienced symptomatic RP. The median interval from the completion of radiotherapy to the diagnosis of RP was 65 days (range, 20-137 days).

\section{Univariate Analysis}

The comparisons of clinical and dosimetric factors between patients with or without symptomatic RP are listed in Table 2 and Supplementary Fig. 1A. For clinical characteristics, univariate analysis showed that age, smoking history, performance status, primary tumor length, and radiotherapy modality were correlated with the development of RP. Among dosimetric parameters, PTV, Lung $V_{5}$ to $V_{30}$, and MLD were 
significantly associated with the risk of $\operatorname{RP}(P<0.05$ for all). Nevertheless, no pulmonary function parameters were significant risk factors for RP. 
Table 2

Univariate analysis for variables associated with symptomatic radiation pneumonitis in the primary cohort $(n=321)$.

\begin{tabular}{|c|c|c|c|c|}
\hline \multirow[t]{2}{*}{ Characteristic } & \multirow{2}{*}{$\begin{array}{l}\text { With RP } \\
(n=31), \%\end{array}$} & \multirow{2}{*}{$\begin{array}{l}\text { Without RP } \\
(n=290), \%\end{array}$} & \multicolumn{2}{|l|}{ Univariate } \\
\hline & & & $\begin{array}{l}\text { Odds ratio (95\% } \\
\mathrm{Cl})\end{array}$ & $\begin{array}{l}P \\
\text { value }\end{array}$ \\
\hline \multicolumn{5}{|l|}{ Clinical factors } \\
\hline \multicolumn{5}{|l|}{ Age (years) } \\
\hline$<59$ & $9(29.0)$ & $147(50.7)$ & Ref & \\
\hline$\geq 59$ & $22(71.0)$ & $143(49.3)$ & $\begin{array}{l}2.513(1.119- \\
5.643)\end{array}$ & 0.026 \\
\hline \multicolumn{5}{|l|}{ Sex } \\
\hline Male & $26(83.9)$ & 219 (75.5) & Ref & \\
\hline Female & $5(16.1)$ & $71(24.5)$ & $\begin{array}{l}0.593(0.220- \\
1.602)\end{array}$ & 0.303 \\
\hline \multicolumn{5}{|c|}{ Smoking history } \\
\hline Yes & $17(54.8)$ & $201(69.3)$ & Ref & \\
\hline No & $14(45.2)$ & $89(30.7)$ & $\begin{array}{l}1.860(0.878- \\
3.938)\end{array}$ & 0.105 \\
\hline \multicolumn{5}{|l|}{ COPD } \\
\hline Yes & $2(6.5)$ & $16(5.5)$ & Ref & \\
\hline No & $29(93.5)$ & $274(94.5)$ & $\begin{array}{l}0.847(0.185- \\
3.867)\end{array}$ & 0.830 \\
\hline \multicolumn{5}{|c|}{ ECOG performance status } \\
\hline 0 & $16(51.6)$ & $199(68.6)$ & Ref & \\
\hline $1-2$ & $15(48.4)$ & $91(31.4)$ & $\begin{array}{l}2.050(0.972- \\
4.326)\end{array}$ & 0.060 \\
\hline \multicolumn{5}{|l|}{ Weight loss } \\
\hline$<10 \%$ & $23(74.2)$ & $231(79.7)$ & Ref & \\
\hline
\end{tabular}

Abbreviations: $\mathrm{RP}$, radiation pneumonitis; $\mathrm{Cl}$, confidence interval; $\mathrm{COPD}$, chronic obstructive pulmonary disease; ECOG, Eastern Cooperative Oncology Group; 3DCRT, three-dimensional conformal radiation therapy; IMRT, intensity-modulated radiation therapy; $\mathrm{V}_{\mathrm{x}}$ : percentage of the total lung volume receiving more than $x$ Gy; MLD, mean lung dose; PTV, planning target volume; TLV, total lung volume; FEV1: forced expiratory volume in the first second; DLCO, diffusing capacity for carbon monoxide.

*: Single agent chemotherapy during radiotherapy (taxane or platinum). 


\begin{tabular}{|c|c|c|c|c|}
\hline \multirow[t]{2}{*}{ Characteristic } & \multirow{2}{*}{$\begin{array}{l}\text { With RP } \\
(n=31), \%\end{array}$} & \multirow{2}{*}{$\begin{array}{l}\text { Without RP } \\
(n=290), \%\end{array}$} & \multicolumn{2}{|l|}{ Univariate } \\
\hline & & & $\begin{array}{l}\text { Odds ratio (95\% } \\
\mathrm{Cl})\end{array}$ & $\begin{array}{l}P \\
\text { value }\end{array}$ \\
\hline$\geq 10 \%$ & $8(25.8)$ & $59(20.3)$ & $\begin{array}{l}1.362(0.580- \\
3.198)\end{array}$ & 0.478 \\
\hline \multicolumn{5}{|l|}{ Tumor location } \\
\hline Upper & $10(32.3)$ & $117(40.3)$ & Ref & \\
\hline Middle & $17(54.8)$ & $132(45.5)$ & $\begin{array}{l}1.320(0.420- \\
4.145)\end{array}$ & 0.634 \\
\hline Distal & $4(12.9)$ & $41(14.1)$ & $\begin{array}{l}0.876(0.260- \\
2.946)\end{array}$ & 0.831 \\
\hline \multicolumn{5}{|l|}{ Primary tumor length } \\
\hline$<6 \mathrm{~cm}$ & $13(41.9)$ & $167(57.6)$ & Ref & \\
\hline$\geq 6 \mathrm{~cm}$ & $18(58.1)$ & $123(42.4)$ & $\begin{array}{l}1.880(0.888- \\
3.982)\end{array}$ & 0.099 \\
\hline \multicolumn{5}{|l|}{ Clinical TNM stage } \\
\hline I/II & $2(6.5)$ & $32(11.0)$ & Ref & \\
\hline III/IVa & $29(93.5)$ & $258(89.0)$ & $\begin{array}{l}1.798(0.410- \\
7.894)\end{array}$ & 0.437 \\
\hline \multicolumn{5}{|c|}{ Induction chemotherapy } \\
\hline Yes & $17(54.8)$ & $178(61.4)$ & Ref & \\
\hline No & $14(45.2)$ & $112(38.6)$ & $\begin{array}{l}1.309(0.621- \\
2.759)\end{array}$ & 0.479 \\
\hline \multicolumn{5}{|l|}{ Chemotherapy regimen } \\
\hline Cisplatin/taxane & $14(45.2)$ & $159(54.8)$ & Ref & \\
\hline Cisplatin/fluorouracil & $6(19.4)$ & $62(21.4)$ & $\begin{array}{l}0.552(0.239- \\
1.278)\end{array}$ & 0.165 \\
\hline Other ${ }^{*}$ & $11(35.5)$ & $69(23.8)$ & $\begin{array}{l}0.607(0.212- \\
1.739)\end{array}$ & 0.352 \\
\hline
\end{tabular}

Abbreviations: RP, radiation pneumonitis; $\mathrm{Cl}$, confidence interval; COPD, chronic obstructive pulmonary disease; ECOG, Eastern Cooperative Oncology Group; 3DCRT, three-dimensional conformal radiation therapy; IMRT, intensity-modulated radiation therapy; $\mathrm{V}_{\mathrm{x}}$ : percentage of the total lung volume receiving more than $x$ Gy; MLD, mean lung dose; PTV, planning target volume; TLV, total lung volume; FEV1: forced expiratory volume in the first second; DLCO, diffusing capacity for carbon monoxide.

*: Single agent chemotherapy during radiotherapy (taxane or platinum). 


\begin{tabular}{|c|c|c|c|c|}
\hline \multirow[t]{2}{*}{ Characteristic } & \multirow{2}{*}{$\begin{array}{l}\text { With RP } \\
(n=31), \%\end{array}$} & \multirow{2}{*}{$\begin{array}{l}\text { Without RP } \\
(n=290), \%\end{array}$} & \multicolumn{2}{|l|}{ Univariate } \\
\hline & & & $\begin{array}{l}\text { Odds ratio (95\% } \\
\mathrm{Cl})\end{array}$ & $\begin{array}{l}P \\
\text { value }\end{array}$ \\
\hline \multicolumn{5}{|l|}{ Radiation dose (Gy) } \\
\hline$\leq 60$ & $22(71.0)$ & $215(74.1)$ & Ref & \\
\hline$>60$ & $9(29.0)$ & $75(25.9)$ & $\begin{array}{l}1.173(0.517- \\
2.660)\end{array}$ & 0.703 \\
\hline \multicolumn{5}{|l|}{ Radiotherapy modality } \\
\hline 3DCRT & $15(48.4)$ & $55(19.0)$ & Ref & \\
\hline IMRT & $16(51.6)$ & $235(81.0)$ & $\begin{array}{l}0.250(0.116- \\
0.535)\end{array}$ & $<.001$ \\
\hline \multicolumn{5}{|l|}{ Dosimetric parameters } \\
\hline $\mathrm{V}_{5}(\%)$, Median (IQR) & $75.9(71.6-89.2)$ & $69.6(59.9-80.9)$ & $\begin{array}{l}1.035(1.009- \\
1.062)\end{array}$ & 0.009 \\
\hline $\mathrm{V}_{10}(\%)$, Median (IQR) & $54.2(48.5-67.3)$ & $48.7(41.5-56.0)$ & $\begin{array}{l}1.042(1.013- \\
1.072)\end{array}$ & 0.005 \\
\hline $\mathrm{V}_{15}(\%)$, Median (IQR) & $40.9(37.2-49.0)$ & $35.6(30.9-40.9)$ & $\begin{array}{l}1.085(1.039- \\
1.134)\end{array}$ & $\begin{array}{l}< \\
0.001\end{array}$ \\
\hline $\mathrm{V}_{20}(\%)$, Median (IQR) & $30.4(28.3-33.1)$ & $26.9(22.3-29.9)$ & $\begin{array}{l}1.175(1.080- \\
1.278)\end{array}$ & $\begin{array}{l}<.001 \\
0.001\end{array}$ \\
\hline $\mathrm{V}_{25}(\%)$, Median (IQR) & $22.4(20.7-23.9)$ & $20.2(16.0-22.7)$ & $\begin{array}{l}1.146(1.049- \\
1.252)\end{array}$ & 0.002 \\
\hline $\mathrm{V}_{30}(\%)$, Median (IQR) & $17.3(13.3-19.8)$ & $14.7(11.2-17.4)$ & $\begin{array}{l}1.143(1.045- \\
1.250)\end{array}$ & 0.003 \\
\hline MLD (Gy), Median (IQR) & $17.0(15.2-18.2)$ & $14.7(12.6-16.5)$ & $\begin{array}{l}1.349(1.158- \\
1.572)\end{array}$ & $<.001$ \\
\hline PTV (cc), Median (IQR) & $\begin{array}{l}772.0(639.0- \\
921.5)\end{array}$ & $\begin{array}{l}665.6(532.7- \\
812.1)\end{array}$ & $\begin{array}{l}1.002(1.000- \\
1.003)\end{array}$ & 0.019 \\
\hline TLV (cc) & $\begin{array}{l}3080.4(2648.5- \\
3938.1)\end{array}$ & $\begin{array}{l}3265.4(2711.0- \\
3895.4)\end{array}$ & $\begin{array}{l}0.693(0.327- \\
1.466)\end{array}$ & 0.717 \\
\hline
\end{tabular}

Abbreviations: $\mathrm{RP}$, radiation pneumonitis; $\mathrm{Cl}$, confidence interval; $\mathrm{COPD}$, chronic obstructive pulmonary disease; ECOG, Eastern Cooperative Oncology Group; 3DCRT, three-dimensional conformal radiation therapy; IMRT, intensity-modulated radiation therapy; $\mathrm{V}_{\mathrm{x}}$ : percentage of the total lung volume receiving more than $x$ Gy; MLD, mean lung dose; PTV, planning target volume; TLV, total lung volume; FEV1: forced expiratory volume in the first second; DLCO, diffusing capacity for carbon monoxide.

*: Single agent chemotherapy during radiotherapy (taxane or platinum). 


\begin{tabular}{|c|c|c|c|c|}
\hline \multirow[t]{2}{*}{ Characteristic } & \multirow{2}{*}{$\begin{array}{l}\text { With RP } \\
(n=31), \%\end{array}$} & \multirow{2}{*}{$\begin{array}{l}\text { Without RP } \\
(n=290), \%\end{array}$} & \multicolumn{2}{|l|}{ Univariate } \\
\hline & & & $\begin{array}{l}\text { Odds ratio }(95 \% \\
\text { Cl) }\end{array}$ & $\begin{array}{l}P \\
\text { value }\end{array}$ \\
\hline \multicolumn{5}{|l|}{$\begin{array}{l}\text { Pulmonary function } \\
\text { parameters }\end{array}$} \\
\hline FEV1 (L), Median (IQR) & $2.7(2.3-3.1)$ & $2.7(1.9-3.2)$ & $\begin{array}{l}0.696(0.336- \\
1.440)\end{array}$ & 0.328 \\
\hline FEV1 (\%), Median (IQR) & 93.7 (78.2-104.8) & $93.5(78.0-103.7)$ & $\begin{array}{l}0.994(0.951- \\
1.040)\end{array}$ & 0.808 \\
\hline $\begin{array}{l}\text { DLCO }(\mathrm{ml} / \mathrm{mmHg} / \mathrm{min}) \\
\text { Median (IQR) }\end{array}$ & $24.2(20.6-27.8)$ & $24.2(20.8-27.9)$ & $\begin{array}{l}1.001(0.910- \\
1.102)\end{array}$ & 0.977 \\
\hline \multicolumn{5}{|c|}{$\begin{array}{l}\text { Abbreviations: RP, radiation pneumonitis; } \mathrm{Cl} \text {, confidence interval; } \mathrm{COPD} \text {, chronic obstructive pulmonary } \\
\text { disease; ECOG, Eastern Cooperative Oncology Group; } 3 \mathrm{D} \text { CRT, three-dimensional conformal radiation } \\
\text { therapy; IMRT, intensity-modulated radiation therapy; } \mathrm{V}_{\mathrm{x}} \text { : percentage of the total lung volume receiving } \\
\text { more than x Gy; MLD, mean lung dose; PTV, planning target volume; TLV, total lung volume; FEV1: } \\
\text { forced expiratory volume in the first second; DLCO, diffusing capacity for carbon monoxide. }\end{array}$} \\
\hline
\end{tabular}

\section{Multivariate Analysis}

Considering the possible relationships between dosimetric parameters of lung, Spearman's correlation analysis was performed and indicated strong correlations between $\mathrm{V}_{10}$ and $\mathrm{V}_{15}(\mathrm{\gamma}=0.901, P<0.01)$, as well as $V_{25}$ and $V_{30}(Y=0.906, P<0.01)$ (Supplementary Table 1$)$. According to the odds ratios $(O R), V_{15}$ instead of $\mathrm{V}_{10}$ and $\mathrm{V}_{25}$ instead of $\mathrm{V}_{30}$ were included in the multivariate analysis to avoid multicollinearity. On multivariate analysis, smoking history (OR: 2.842, $P=0.018$ ), radiotherapy modality (OR: $5.294, P<$ 0.001), PTV (OR: $1.002, P=0.014)$, and lung $\mathrm{V}_{20}$ (OR: 1.147, $\left.P=0.002\right)$ were independent predictive factors for symptomatic RP (Table 3). Among these factors, smoking history was found to protect against RP. 
Table 3

Multivariate and ROC analysis for variables associated with symptomatic radiation pneumonitis in the primary cohort.

\begin{tabular}{|c|c|c|c|c|}
\hline \multirow[t]{2}{*}{ Variable } & \multicolumn{2}{|l|}{ Multivariate analysis } & \multicolumn{2}{|l|}{ ROC curve } \\
\hline & Odds ratio (95\% Cl) & $\begin{array}{l}P \\
\text { value }\end{array}$ & $\operatorname{AUC}(95 \% \mathrm{Cl})$ & $\begin{array}{l}P \\
\text { value }\end{array}$ \\
\hline Smoking history (no vs. yes) & $\begin{array}{l}2.842(1.195- \\
6.756)\end{array}$ & 0.018 & $\begin{array}{l}0.572(0.463- \\
0.681)\end{array}$ & 0.008 \\
\hline $\begin{array}{l}\text { Radiotherapy modality (3DCRT vs. } \\
\text { IMRT) }\end{array}$ & $\begin{array}{l}5.294(2.202- \\
12.725)\end{array}$ & $<001$ & $\begin{array}{l}0.647(0.536- \\
0.758)\end{array}$ & 0.096 \\
\hline PTV & $\begin{array}{l}1.002(1.000- \\
1.004)\end{array}$ & 0.014 & $\begin{array}{l}0.724(0.632- \\
0.816)\end{array}$ & 0.484 \\
\hline Lung $\mathrm{V}_{20}$ & $\begin{array}{l}1.147(1.053- \\
1.250)\end{array}$ & 0.002 & $\begin{array}{l}0.628(0.529- \\
0.727)\end{array}$ & 0.042 \\
\hline Nomogram & / & / & $\begin{array}{l}0.772(0.673- \\
0.871)\end{array}$ & Ref. \\
\hline \multicolumn{5}{|c|}{$\begin{array}{l}\text { Abbreviations: ROC, receiver operating characteristic; } \mathrm{Cl} \text {, confidence interval; } \mathrm{AUC} \text {, area under the } \\
\text { curve; 3DCRT, three-dimensional conformal radiation therapy; IMRT, intensity-modulated radiation } \\
\text { therapy; PTV, planning target volume; } \mathrm{V}_{20} \text { : percentage of the total lung volume receiving more than } \\
20 \mathrm{~Gy} \text {. }\end{array}$} \\
\hline
\end{tabular}

Figure 1 shows the predictive probabilities of symptomatic RP as a function of PTV or lung $\mathrm{V}_{20}$ for the two radiation modalities based on logistic regression model. Patients with larger PTVs or higher lung $V_{20}$ had a higher predictive risk of RP, whereas the overall risk was substantially higher for 3DCRT than IMRT. For example, when PTV is $630.0 \mathrm{cc}$, the probability of developing symptomatic RP would be $5.0 \%$ in patients treated with IMRT versus $20.2 \%$ in those with 3DCRT.

\section{Nomogram Development and Validation}

Based on the multivariate analysis, a nomogram model was built to predict the risk of symptomatic RP, including smoking history, radiotherapy modality, PTV, and lung $\mathrm{V}_{20}$ (Fig. 2). The AUC of predictive model was 0.772 (95\% Cl: $0.673-0.871$ ), which was superior to each predictor alone (smoking history: 0.572 , 95\% Cl: 0.463-0.681; radiotherapy modality: 0.647 , 95\% Cl: 0.536-0.758; PTV: $0.724,95 \%$ Cl: $0.632-$ 0.816 ; and lung $\mathrm{V}_{20}: 0.628,95 \% \mathrm{Cl}: 0.529-0.727$ ) (Fig. 3A). The optimal cut-offs for PTV and lung $\mathrm{V}_{20}$ were $713.0 \mathrm{cc}$ and $27.5 \%$, respectively. Additionally, the calibration curve showed favorable agreement between the prediction by nomogram and the actual observation (Fig. 3B). Also, the DCA exhibited satisfactory positive net benefits of the model among the majority of threshold probabilities, indicating excellent clinical utility (Fig. 3C).

In the validation cohort $(n=81), 10$ patients $(12.3 \%)$ experienced grade $\geq 2$ RP. The comparisons of lung dosimetric factors between patients with or without symptomatic RP are listed in Supplementary Fig. 1B. 
Application of the nomogram model in the validation cohort yielded an excellent AUC of $0.900(95 \% \mathrm{Cl}$ : 0.779-1.000) (Supplementary Table 2 and Supplementary Fig. 2).

\section{Discussion}

Symptomatic RP is a common side effect of radiotherapy for EC with incidence of 5.7-35.0\% [5-11], which is confirmed by our study. In this cohort of ESCC patients undergoing definitive CRT, smoking history, radiotherapy modality, PTV, and lung $\mathrm{V}_{20}$ were significant predictive factors for symptomatic RP. More importantly, a nomogram has been built and validated, indicating satisfactory prediction ability. Thus, this prediction model could help clinicians select high-risk patients who may benefit from modified treatment approaches to reduce the risk of RP prior to the initiation of treatment.

In recent years, radiation techniques have evolved from 3DCRT to IMRT and proton therapy in EC. Numerous dosimetric studies have well demonstrated the superiority of IMRT over 3DCRT in improving target coverage and sparing adjacent organs, but whether the dosimetric benefits could translate into clinical benefit, especially reducing the incidence of radiation-related toxicities, remains inconclusive due to the lack of prospective evidence [14]. In a large-scale retrospective study reported by He et al, IMRT significantly reduced the incidence and postponed the onset of pleural effusion in EC patients, compared to 3DCRT [15]. However, Haefner et al found no evident difference in acute toxicities between IMRT and 3DCRT, possibly due to the small sample size of the cohort and the higher radiation dose in the IMRT group [16]. In our study, after adjusting for smoking history, PTV, and Lung $\mathrm{V}_{20}$, IMRT was associated with a substantially lower risk of RP than 3DCRT. This is in consistence with the secondary analysis of results from RTOG 0617, which prospectively demonstrated that IMRT group had significantly less severe RP than 3DCRT group in locally advanced non-small cell lung cancer (3.5\% vs. $7.9 \%, P=0.039)$ [17]. In addition, IMRT could also reduce the incidence of postoperative pulmonary and cardiac complication in EC patients who received neoadjuvant CRT and surgery, as reported by Lin et al [18]. Furthermore, IMRT was associated with more favorable survival outcomes than 3DCRT in EC [14]. Collectively, despite the paucity of prospective evidence, the current findings strongly suggest the routine use of IMRT in EC.

As a novel radiation technique with superior physical properties, proton therapy has the potential to improve normal tissue sparing as compared to 3DCRT or IMRT [14]. Several studies have investigated the clinical advantages of proton therapy compared to photon therapy. For EC patients who underwent neoadjuvant CRT, proton therapy was superior to IMRT in reducing incidence of pulmonary complications [18]. Recently, Lin et al reported that the proton arm experienced numerically fewer cardiopulmonary toxicities compared with IMRT arm in a phase IIB randomized trial for EC [19]. The ongoing larger cooperative group studies will clarify the clinical benefit of proton therapy in EC, such as NRG-GI006.

There has been a general consensus that DVH parameters are important predictors for RP. However, there is still no recommendation of dose-volume constraints for EC. Wang et al reported that the volume of the

lung spared from doses of $\geq 5$ Gy was the only independent dosimetric factor associated with pulmonary complications [20]. Cho et al indicated that MLD was the parameter most related to pulmonary 
complications in EC [21]. Likewise, a recent study demonstrated the strong correlation between MLD and severe RP in 416 EC patients undergoing CRT [22]. Consistent with studies reported by Asakura et al and Shaikh et al [9, 23], all DVH parameters (lung $\mathrm{V}_{5}-\mathrm{V}_{30}$ and MLD) were significantly associated with RP in univariate analysis in our cohort. Of them, $\mathrm{V}_{20}$ was the only independent predictor in multivariate analysis, with the optimal threshold value of $\mathrm{V}_{20}(27.5 \%)$. The failure of the other dosimetric parameters to be retained significance in the multivariate model could be explained by their potential correlation with $\mathrm{V}_{20}$. Thus, other dosimetric parameters should also be taken into consideration when performing treatment planning for EC.

In addition to lung dosimetric parameters, we observed that patients with greater PTVs had a remarkably higher incidence of RP. In line with our results, Cui et al also reported the strong correlation between PTV and the occurrence of RP in elderly EC [24]. Considering PTV is a variable that could be modified, smaller radiation volumes might reduce the risk of RP. A meta-analysis reported that neither local control rates nor survival outcomes differed significantly between elective nodal irradiation and involved-field irradiation in ESCC, whereas incidences of severe RP and radiation esophagitis were significantly lower in the latter group [25]. Therefore, involved-field irradiation should be considered in clinical practice, especially for elderly patients. Among clinical factors, smoking history was found to be a protective factor for RP in our study, which is consistent with previous studies $[26,27]$. The possible explanations are smokingassociated hypoxia and a decreased inflammatory reaction induced by irradiation among smokers [28, 29], but the underlying mechanism for the smoking effect on RP remains unclear.

Compared with each separate predictor, integrating predictive factors to develop a statistical model will further improve predictive accuracy. So far, only one study reported by Wang et al had built a combined model to predict severe RP in EC [22]. However, this study included a fraction of patients without concurrent chemotherapy during radiotherapy, which might affect the incidence of RP. Moreover, this study incorporated not only pretreatment factors but also the changes of inflammatory indexes during radiotherapy into the nomogram [22]. Despite excellent discriminatory power of the model, it is of less value to guide decision-making before the initiation of treatment.

It should be noted that our work also has several limitations. Firstly, selection bias existed due to the retrospective nature of this study. Secondly, the sample size of the validation set is relatively small, and an independent external validation is needed. Thirdly, although all patients included were treated with concurrent CRT, treatment modalities such as the utility of induction chemotherapy and chemotherapy regimens were not identical in this study. However, these factors were not correlated with the risk of RP in univariate analysis. Finally, serum biomarkers as well as radiomics-based features were not incorporated into the study, which might further promote predictive ability of the model.

\section{Conclusions}

Non-smoking status, 3DCRT, lung $\mathrm{V}_{20}(>27.5 \%)$, and PTV $(\geq 713.0 \mathrm{cc})$ were significantly associated with a higher risk of symptomatic RP in ESCC patients treated with definitive CRT. Then a nomogram was built 
and validated, exhibiting satisfactory prediction ability. Further studies are warranted to verify the efficacy of the predicting model and to explore potential strategies for minimizing the risk of RP in high-risk patients.

\section{Abbreviations}

AUC: Area Under the Curve; Cl: Confidence Interval; COPD: Chronic Obstructive Pulmonary Disease; CRT: Definitive Chemoradiotherapy; CTCAE: National Cancer Institute Common Toxicity Criteria for Adverse Events; CTV: Clinical Target Volume; DCA: Decision Curve Analysis; DLCO: Diffusing Capacity for Carbon Monoxide; DVH: Dose-Volume Histogram; EC: Esophageal Cancer; ECOG: Eastern Cooperative Oncology Group; ESCC: Esophageal Squamous Cell Carcinoma; FEV1: Forced Expiratory Volume in the First Second; GTV: Gross Tumor Volume; IMRT: Intensity-Modulated Radiotherapy; MLD: Mean Lung Dose; PTV: Planning Target Volume; ROC: Receiver Operating Characteristic; RP: Radiation Pneumonitis; TLV: Total Lung Volume; Vx: Percentage of the Total Lung Volume Receiving more than x Gy; 3DCRT: ThreeDimensional Conformal Radiotherapy.

\section{Declarations}

\section{Ethics approval and consent to participate}

The study was approved by our institution.

\section{Consent for publication}

Not applicable.

\section{Availability of data and materials}

All data generated or analysed during this study were included in this published article.

\section{Competing interests}

The authors declare that there is no conflict of interest.

\section{Funding}

This work was supported by grants from the Fundamental Research Funds for the Central Universities (19ykpy176) and Natural Science Foundation of Guangdong Province (2019A1515011420). 


\section{Author contributions}

Conception and design: LZ, MX.

Acquisition of data: KQL, CX, SLL, JHZ, YDY, LZ, MX.

Analysis and interpretation of data: KQL, CX, SLL, JHZ, YDY, LZ, MX.

Writing, review, and/or revision of the manuscript: KQL, CX, SLL, JHZ, YDY, LZ, MX.

All authors read and approved the final manuscript.

\section{Acknowledgements}

We thank all the patients who participated in this study.

\section{Author details}

${ }^{1}$ State Key Laboratory of Oncology in South China, Collaborative Innovation Centre for Cancer Medicine, Guangdong Esophageal Cancer Institute, Guangzhou, China;

${ }^{2}$ Department of Nasopharyngeal Carcinoma, Sun Yat-sen University Cancer Center, Guangzhou, China;

${ }^{3}$ Department of Radiation Oncology, Sun Yat-sen University Cancer Center, Guangzhou, China;

${ }^{4}$ Department of Imaging Diagnosis and Interventional Center, Sun Yat-sen University Cancer Center, Guangzhou, China.

\section{References}

1. Bray F, Ferlay J, Soerjomataram I, et al. Global cancer statistics 2018: GLOBOCAN estimates of incidence and mortality worldwide for 36 cancers in 185 countries. CA Cancer J Clin. 2018;68(6):394424.

2. Cooper JS, Guo MD, Herskovic A, et al. Chemoradiotherapy of locally advanced esophageal cancer: long-term follow-up of a prospective randomized trial (RTOG 85-01). Radiation Therapy Oncology Group. JAMA. 1999;281(17):1623-7.

3. Conroy T, Galais M-P, Raoul J-L, et al. Definitive chemoradiotherapy with FOLFOX versus fluorouracil and cisplatin in patients with oesophageal cancer (PRODIGE5/ACCORD17): final results of a randomised, phase 2/3 trial. The Lancet Oncology. 2014;15(3):305-314.

4. Hanania AN, Mainwaring W, Ghebre YT, et al. Radiation-Induced Lung Injury: Assessment and Management. Chest. 2019;156(1):150-162. 
5. McCurdy M, McAleer MF, Wei W, et al. Induction and concurrent taxanes enhance both the pulmonary metabolic radiation response and the radiation pneumonitis response in patients with esophagus cancer. Int J Radiat Oncol Biol Phys. 2010;76(3):816-23.

6. Zhao L, Ji W, Ou G, et al. Risk factors for radiation-induced lung toxicity in patients with non-small cell lung cancer who received postoperative radiation therapy. Lung Cancer. 2012;77(2):326-30.

7. Zhang $L$, Yang $M, B i N$, et al. ATM polymorphisms are associated with risk of radiation-induced pneumonitis. Int J Radiat Oncol Biol Phys. 2010;77(5):1360-8.

8. Lu SL, Hsu FM, Tsai CL, et al. Serum Transforming Growth Factor-beta1 Change After Neoadjuvant Chemoradiation Therapy Is Associated With Postoperative Pulmonary Complications in Esophageal Cancer Patients Undergoing Combined Modality Therapy. Int J Radiat Oncol Biol Phys. 2015;93(5):1023-31.

9. Asakura $\mathrm{H}$, Hashimoto $\mathrm{T}$, Zenda $\mathrm{S}$, et al. Analysis of dose-volume histogram parameters for radiation pneumonitis after definitive concurrent chemoradiotherapy for esophageal cancer. Radiother Oncol. 2010;95(2):240-4.

10. Wang S, Liao Z, Wei X, et al. Association between systemic chemotherapy before chemoradiation and increased risk of treatment-related pneumonitis in esophageal cancer patients treated with definitive chemoradiotherapy. J Thorac Oncol. 2008;3(3):277-82.

11. Nomura $\mathrm{M}$, Kodaira T, Furutani $\mathrm{K}$, et al. Predictive factors for radiation pneumonitis in oesophageal cancer patients treated with chemoradiotherapy without prophylactic nodal irradiation. $\mathrm{Br} \mathrm{J}$ Radiol. 2012;85(1014):813-8.

12. Ueyama T, Arimura T, Takumi K, et al. Risk factors for radiation pneumonitis after stereotactic radiation therapy for lung tumours: clinical usefulness of the planning target volume to total lung volume ratio. $\mathrm{Br} J$ Radiol. 2018;91(1086):20170453.

13. Rice TW, Ishwaran $H$, Blackstone EH, et al. Recommendations for clinical staging (cTNM) of cancer of the esophagus and esophagogastric junction for the 8th edition AJCC/UICC staging manuals. Dis Esophagus. 2016;29(8):913-919.

14. Xi M, Lin SH. Recent advances in intensity modulated radiotherapy and proton therapy for esophageal cancer. Expert Rev Anticancer Ther. 2017;17(7):635-646.

15. He L, Chapple A, Liao Z, et al. Bayesian regression analyses of radiation modality effects on pericardial and pleural effusion and survival in esophageal cancer. Radiother Oncol 2016;121(1):7074.

16. Haefner MF, Lang K, Verma V, et al. Intensity-modulated versus 3-dimensional conformal radiotherapy in the definitive treatment of esophageal cancer: comparison of outcomes and acute toxicity. Radiat Oncol. 2017;12(1):131.

17. Chun SG, Hu C, Choy H, et al. Impact of Intensity-Modulated Radiation Therapy Technique for Locally Advanced Non-Small-Cell Lung Cancer: A Secondary Analysis of the NRG Oncology RTOG 0617 Randomized Clinical Trial. J Clin Oncol. 2017;35(1):56-62. 
18. Lin SH, Merrell KW, Shen J, et al. Multi-institutional analysis of radiation modality use and postoperative outcomes of neoadjuvant chemoradiation for esophageal cancer. Radiother Oncol. 2017;123(3):376-381.

19. Lin SH, Hobbs BP, Verma V, et al. Randomized Phase IIB Trial of Proton Beam Therapy Versus Intensity-Modulated Radiation Therapy for Locally Advanced Esophageal Cancer. J Clin Oncol. 2020;38(14):1569-1579.

20. Wang SL, Liao Z, Vaporciyan AA, et al. Investigation of clinical and dosimetric factors associated with postoperative pulmonary complications in esophageal cancer patients treated with concurrent chemoradiotherapy followed by surgery. Int J Radiat Oncol Biol Phys. 2006;64(3):692-9.

21. Cho WK, Oh D, Kim HK, et al. Dosimetric predictors for postoperative pulmonary complications in esophageal cancer following neoadjuvant chemoradiotherapy and surgery. Radiother Oncol. 2019;133:87-92.

22. Wang L, Liang S, Li C, et al. A Novel Nomogram and Risk Classification System Predicting Radiation Pneumonitis in Patients With Esophageal Cancer Receiving Radiation Therapy. Int J Radiat Oncol Biol Phys. 2019;105(5):1074-1085.

23. Shaikh T, Churilla TM, Monpara P, et al. Risk of radiation pneumonitis in patients receiving taxanebased trimodality therapy for locally advanced esophageal cancer. Pract Radiat Oncol. 2016;6(6):388-394.

24. Cui Z, Tian Y, He B, et al. Associated factors of radiation pneumonitis induced by precise radiotherapy in 186 elderly patients with esophageal cancer. Int J Clin Exp Med. 2015;8(9):16646-51.

25. Cheng YJ, Jing SW, Zhu LL, et al. Comparison of elective nodal irradiation and involved-field irradiation in esophageal squamous cell carcinoma: a meta-analysis. J Radiat Res. 2018;59(5):604615.

26. Jin H, Tucker SL, Liu HH, et al. Dose-volume thresholds and smoking status for the risk of treatmentrelated pneumonitis in inoperable non-small cell lung cancer treated with definitive radiotherapy. Radiother Oncol. 2009;91(3):427-32.

27. Vogelius IR, Bentzen SM. A literature-based meta-analysis of clinical risk factors for development of radiation induced pneumonitis. Acta Oncol. 2012;51(8):975-83.

28. Bjermer L, Franzen L, Littbrand B, et al. Effects of smoking and irradiated volume on inflammatory response in the lung of irradiated breast cancer patients evaluated with bronchoalveolar lavage. Cancer Res. 1990;50(7):2027-30.

29. Sun S, Schiller JH, Gazdar AF. Lung cancer in never smokers-a different disease. Nat Rev Cancer. 2007;7(10):778-90.

\section{Figures}


A

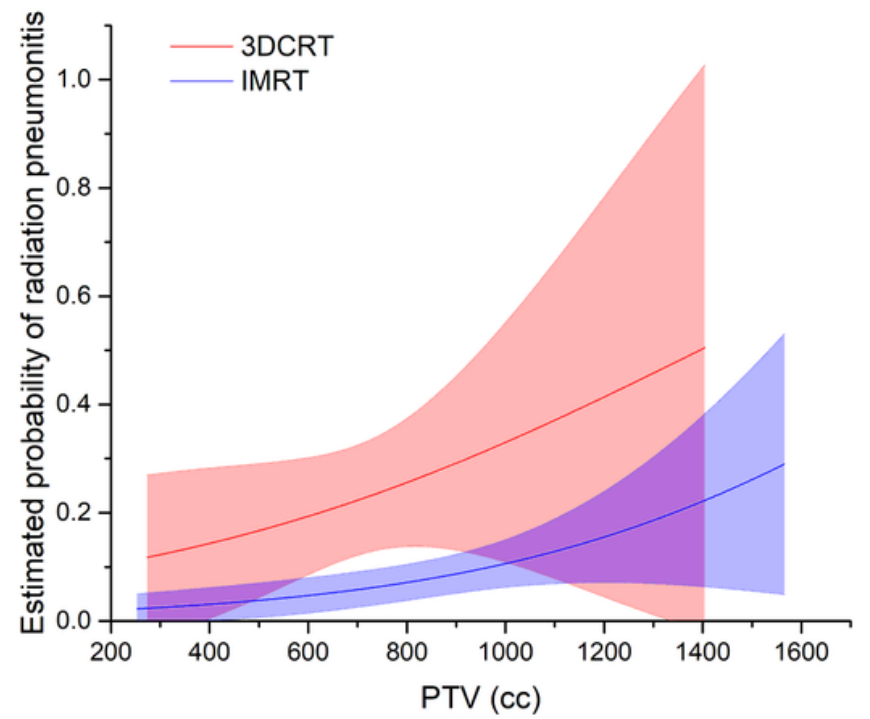

B

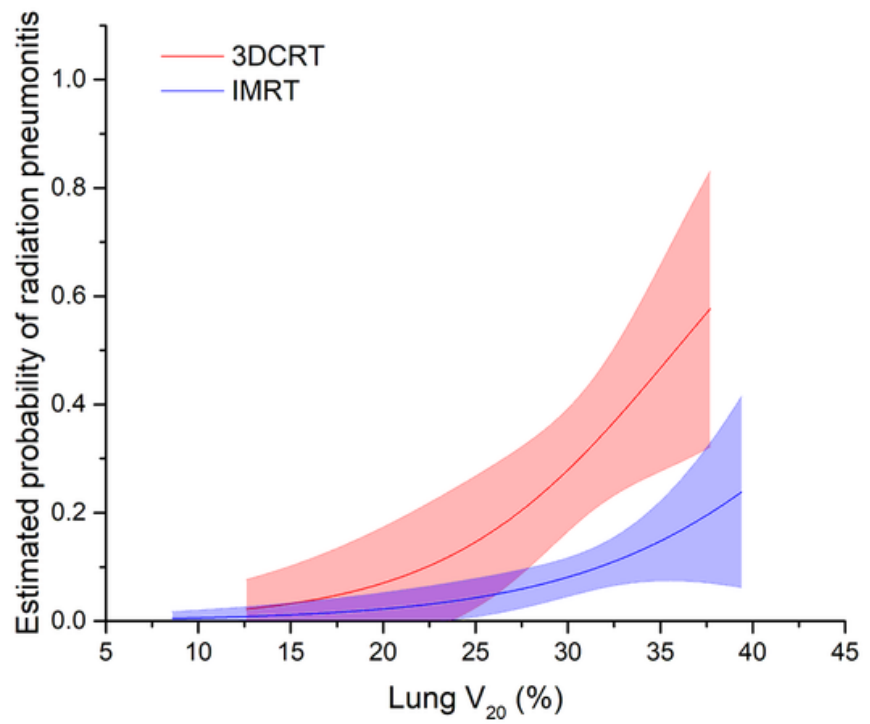

\section{Figure 1}

Model to represent the estimated risk of symptomatic radiation pneumonitis as a function of planning target volume (A) and lung V20 (B) for the two radiation modalities based on logistic regression model in the primary cohort. Red line represents three-dimensional conformal radiotherapy (3DCRT) and blue line represents intensity-modulated radiotherapy (IMRT). Shaded regions represent 95\% point-wise confidence intervals.

Points

0 10 20 30 40 50 60 70 80 90 100

Smoking history

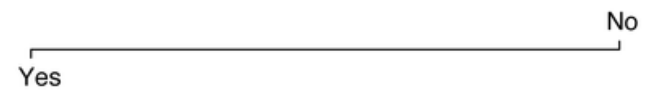

\section{Radiotherapy modality}

3DCRT

PTV (cc)

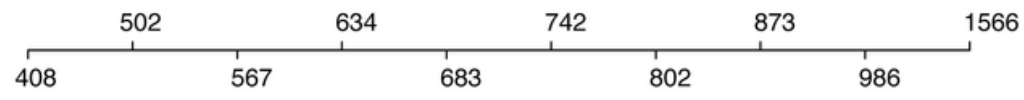

Lung $V_{20}$ (\%)

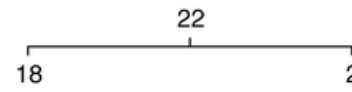

26

28

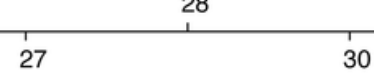

31

30

Total Points

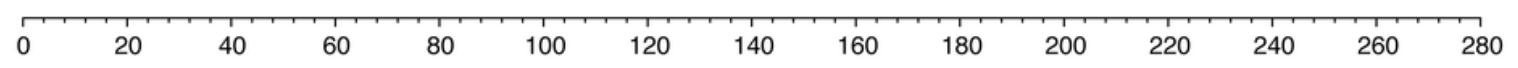

Risk

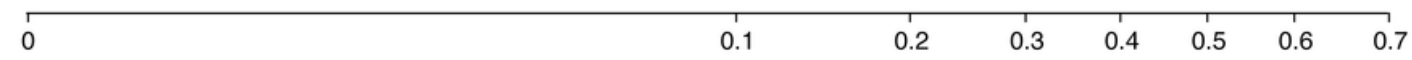


Figure 2

Nomogram predicting the risk of symptomatic radiation pneumonitis in patients with esophageal squamous cell carcinoma who received definitive chemoradiotherapy. For each individual patient, four lines are drawn upward to determine the points received from the four variables in the nomogram. The sum of these points is located on the "Total Points" axis, then a line is drawn downward to predict the risk.
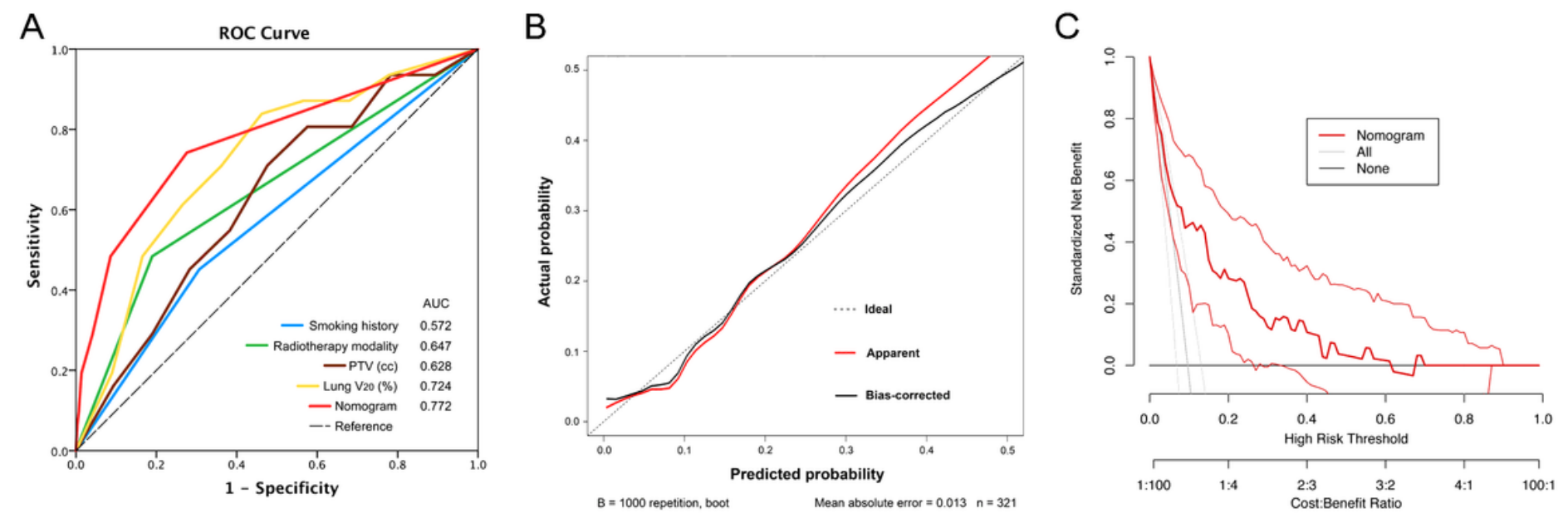

\section{Figure 3}

(A) ROC curves of smoking history, radiotherapy modality, PTV, lung V20, and the nomogram in the primary cohort. (B) Calibration curve of the nomogram predicting the risk of symptomatic radiation pneumonitis in the primary cohort. The $x$-axis and $y$-axis indicate the predicted and actual probabilities of radiation pneumonitis, respectively. (C) Decision curves of the nomogram predicting the risk of radiation pneumonitis in the primary cohort. The x-axis and y-axis show the threshold probabilities and the net benefit, respectively.

\section{Supplementary Files}

This is a list of supplementary files associated with this preprint. Click to download.

- FigureS1.tif

- FigureS2.tif

- Supplementarymaterials.doc 